\title{
Applications of radiocarbon measurements in environmental studies at INFN-LABEC, Florence
}

\author{
M. Fedi ${ }^{1, *}$, P. Álvarez-Iglesias ${ }^{2}$, L. Caforio ${ }^{1,3}$, G. Calzolai $^{1,4}$, \\ V. Bernardoni ${ }^{5}$, M. ChiAri ${ }^{1}$, S. NAVA ${ }^{1}$, \\ F. TACCETTI ${ }^{1}$ and R. VeCCHI $^{5}$
}

${ }^{1}$ INFN, Sezione di Firenze - via Sansone 1, 50019 Sesto Fiorentino (FI) Italy

${ }^{2}$ Departamento de Geociencias Marinas y Ordenación del Territorio Universidad de Vigo - 36310 Vigo, Spain

${ }^{3}$ Dipartimento di Fisica, Università di Ferrara - via Saragat 1 44100 Ferrara, Italy

${ }^{4}$ Dipartimento di Fisica, Università di Firenze - via Sansone 1 50019 Sesto Fiorentino (FI), Italy

${ }^{5}$ INFN, Sezione di Milano e Dipartimento di Fisica dell'Università Milano, Italy

\begin{abstract}
Radiocarbon is one of the most widespread radionuclides in nature. Although it is probably best known for dating in archaeology, in the case of the general public, it represents a useful tracer to study our environment, both in the past and nowadays. For instance, carbonaceous particles, which are in many cases the most abundant among aerosols constituents, are believed to play a major role in both health and climatic effects of aerosols. In particular, measurement of radiocarbon concentration in particulate matter samples can give information on the contributions of the fossil fuels combustion and of natural sources
\end{abstract}

${ }^{*}$ E-mail: fedi@fi.infn.it

This is an Open Access article distributed under the terms of the Creative Commons Attribution License 2.0, which permits unrestricted use, distribution, and reproduction in any medium, provided the original work is properly cited. 
to the carbonaceous fraction in aerosols. These measurements are especially effective when separately performed on different carbonaceous fractions, like elemental and organic carbon (EC and OC, respectively). Past climate is also studied thanks to old archives, as e.g. marine sediments can be. In this case, instead of radiocarbon dating the bulk sediment, a reliable method to fix chronological markers is represented by dating foraminifera tests of $\mathrm{CaCO}_{3}$ picked from different layers in the sediment. Both the aforementioned applications are characterized by the fact that the samples that can be collected for ${ }^{14} \mathrm{C}$ measurements are typically very small, i.e. few $\mathrm{mg}$ or less (before any treatment). Accelerator Mass Spectrometry (AMS) is thus the only technique that can be applied to measure radiocarbon in such samples. Anyway, measurements cannot be so straightforward. In the case of the measurement of radiocarbon concentration in aerosol samples, a preparation line especially dedicated to the extraction of only the carbonaceous fraction of interest is mandatory. Actually, this line should include a combustion oven, from which either total carbon or EC and OC can separately evolve, and a system of traps to purify and collect the $\mathrm{CO}_{2}$. In the case of foraminifera tests (inorganic carbon), special care must be taken in the pre-treatment phase: foraminifera can be contaminated by heterogeneous materials, including organic matter too. Pre-treatment should thus remove all the possible contaminations without losing too much mass of the samples. Here we present an overview of the environmental radiocarbon applications the INFN-LABEC laboratory in Florence is involved in. ${ }^{14} \mathrm{C}$ is measured by AMS, using the dedicated beam line installed at the $3 \mathrm{MV}$ Tandem accelerator. In particular, details about the hardware and the experimental procedures are given.

\section{Introduction}

It is well known that carbon is one of the fundamental constituents of the organic and the inorganic matter on earth. It is present in the atmosphere, primarily as carbon dioxide, in the oceans, for instance as dissolved inorganic carbon, and in the biosphere, as part of molecules on which all living organisms are based. The study of the abundance of the three natural carbon isotopes $\left({ }^{12} \mathrm{C},{ }^{13} \mathrm{C}\right.$ and $\left.{ }^{14} \mathrm{C}\right)$ allows us to investigate many natural processes. In particular, among them, only ${ }^{14} \mathrm{C}$ is radioactive: it decays via $\beta^{-}$-emission to ${ }^{14} \mathrm{~N}$ with a half-life of 5730 years [1]. The equilibrium condition that is established between the formation or the uptake rates and the decaying rate makes it possible that in any living organism the concentration of ${ }^{14} \mathrm{C}$ can be considered stable within time and of the same amount, as a first approximation, as the concentration in atmosphere. The death of the organism 
leads to the end of this equilibrium condition, so that the ${ }^{14} \mathrm{C}$ concentration starts to decrease due to the radioactive exponential decay. Since the 1950's, this mechanism has been exploited to date organic samples taken from an originally living organism (either plants or animals). Actually, radiocarbon has become the most used and powerful natural chronometer to study the past. It has indeed been applied especially to archaeological issues; however, the importance of the ${ }^{14} \mathrm{C}$ chronometer to study the environment is well known as well. An example of this kind of environmental applications is represented by the dating of natural archives [2]. Each archive, e.g. marine sediments, can typically provide proxies, e.g. $\delta^{18} \mathrm{O}$ or $\delta^{13} \mathrm{C}$, which can allow us to reconstruct the environmental changes in the past, i.e. the palaeoclimate. It is evident that associating these proxies with reliable time scales is a crucial point in the discussion about past climate. This can be achieved by radiocarbon [3].

In order to study the present, ${ }^{14} \mathrm{C}$ can also be used as a tracer of anthropogenic activities, since carbon from a fossil origin can be easily distinguished from "modern" carbon: fossil carbon is so old that the ${ }^{14} \mathrm{C}$ content is well below the sensitivity limit of the techniques that we can use to measure it. In other words, ${ }^{14} \mathrm{C}$ content is virtually zero: actually, we often refer to fossil carbon as "dead". An example of ${ }^{14} \mathrm{C}$ used as tracer is given by the measurement of the radiocarbon concentration of the carbonaceous fraction of the particulate matter (PM) in the atmospheric aerosol. PM is known to have strong influence on human health and on environmental changes, although the latter is not well understood yet [4]. Thus, the quantification of the contributions of the different aerosol sources to the aerosol burden in atmosphere, for instance by disentangling the contribution of human activity, is very important for the scientific understanding of the role of the aerosol in the environmental issues and for the development of suited pollution abatement strategies.

Both the aforementioned applications (the dating of marine sediments and the carbon source apportionment studies) are characterized by the fact that the samples to be measured are small, few mg at maximum (see following paragraphs for details). In these cases, the only technique that can be successfully used to measure ${ }^{14} \mathrm{C}$ is Accelerator Mass Spectrometry (AMS). Thanks to the high isobars suppression capabilities due to the use of a Tandem accelerator, Accelerator Mass Spectrometry allows us to reach a very high selective sensitivity even in the case of very small samples: indeed samples as small as few tens of $\mu \mathrm{g}$ carbon can be measured [5].

This paper will discuss the application of ${ }^{14} \mathrm{C}$-AMS to both marine sediments dating and particulate matter measurements, focusing on the activity 


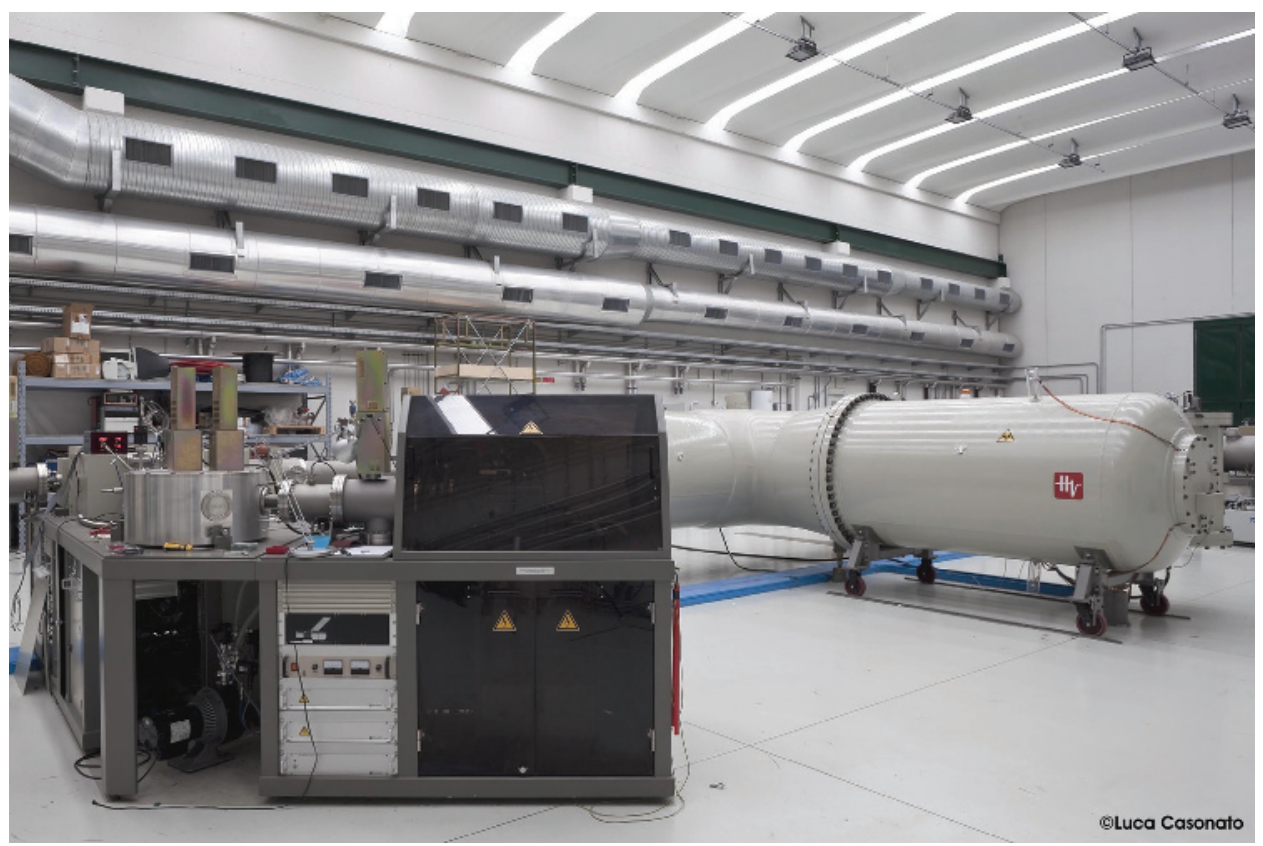

Figure 1: View of the AMS-dedicated ion source and the accelerator of INFNLABEC.

of INFN-LABEC, Florence. The main equipment of LABEC is a $3 \mathrm{MV}$ Tandem accelerator; six beam lines have been installed so far: one is dedicated to AMS [6], the others are dedicated to Ion Beam Analysis measurements (see for example [7-9]).

\section{The ${ }^{14} \mathrm{C}-\mathrm{AMS}$ set-up at INFN-LABEC}

The AMS beam line set-up at INFN-LABEC can be ideally divided into three sections: the low energy side, the accelerator itself and the high energy side.

On the low energy side, a multi-sample Cs-sputtering ion source is installed (see figure 1). This source allows us to extract negative ions from solid samples; in the case of radiocarbon measurements, graphite pellets are inserted into the source (details about the hardware and the procedures to convert samples to be measured into graphite will be given in the following paragraphs). The extracted beam is firstly analysed by passing through an electrostatic analyser according to its energy/charge ratio; typical beam current measured by an insertable Faraday cup after the electrostatic analysis 
is about $15-20 \mu \mathrm{A}$. Then, the beam passes through a magnet to selectively inject into the accelerator the three carbon masses, 12, 13 and 14. To achieve a fast sequential injection, a "bouncer" mechanism is exploited: the magnetic field is kept fixed to transmit a mass, for instance the 13, then, a positive $(\sim 2.9 \mathrm{kV})$ or a negative $(\sim-2.5 \mathrm{kV})$ voltage is applied to the electrically insulated magnet chamber to transmit, respectively, mass 12 and mass 14. In our set-up, each injection cycle lasts for about $10 \mathrm{~ms}$, including $8.5 \mathrm{~ms}, 600 \mu \mathrm{s}$ and $6 \mu \mathrm{s}$ for the transmission of mass 14 , mass 13 and mass 12 , respectively, and the "waiting" time windows between each transition.

Radiocarbon measurements are typically performed at $2.5 \mathrm{MV}$ terminal voltage. At the terminal, the tandem fundamental mechanism of stripping is achieved using Argon gas as the interaction medium. In these conditions, after stripping, the most abundant charge state for the ions is the $3+$ and it's just this charge state the one chosen in the subsequent beam analysis on the high energy side of the AMS beam line.

On the high energy side, the first selective element is a magnet set to transmit ions whose characteristics are: mass 14 , i.e. only ${ }^{14} \mathrm{C}$ since the occurrence of molecular isobars should have been suppressed thanks to stripping, charge state 3 , as absolute value, and thus energy of about $10 \mathrm{MeV}$. At the exit of this magnet, stable isotopes ${ }^{12} \mathrm{C}^{3+}$ and ${ }^{13} \mathrm{C}^{3+}$ are collected by two offset Faraday cups; measured ${ }^{12} \mathrm{C}^{3+}$ and ${ }^{13} \mathrm{C}^{3+}$ average currents (with bouncing on) are typically of the order of a few nA. Signals from the ${ }^{13} \mathrm{C}$-dedicated Faraday cup are also used as a feedback for the stabilization of the terminal voltage. The cup is divided in two sides and the measurement of the balance between the charges collected on each of the sides gives us the information about the trajectory of the ions inside the magnet. As soon as the measured balance appreciably moves from the zero value, the terminal voltage is corrected in order to keep the beam passing through the magnet. Another characteristic of this Faraday cup is the mounting on a movable mechanism, so that its position can be arranged according to the stable isotope and thus to the rare isotope other than ${ }^{14} \mathrm{C}$ we would like to measure.

The analysing magnet is followed by an electrostatic analyser to remove any residual background due to different atomic species with various combinations of mass, energy and charge state, so that they might be transmitted through the analysing magnet. In principle, these ions might be generated, for example, in charge-exchange processes occurring outside the high voltage terminal, along the acceleration tubes.

Downstream the electrostatic analyser (see figure 2), the beam line has been recently upgraded by introducing a home-designed multiwire propor- 


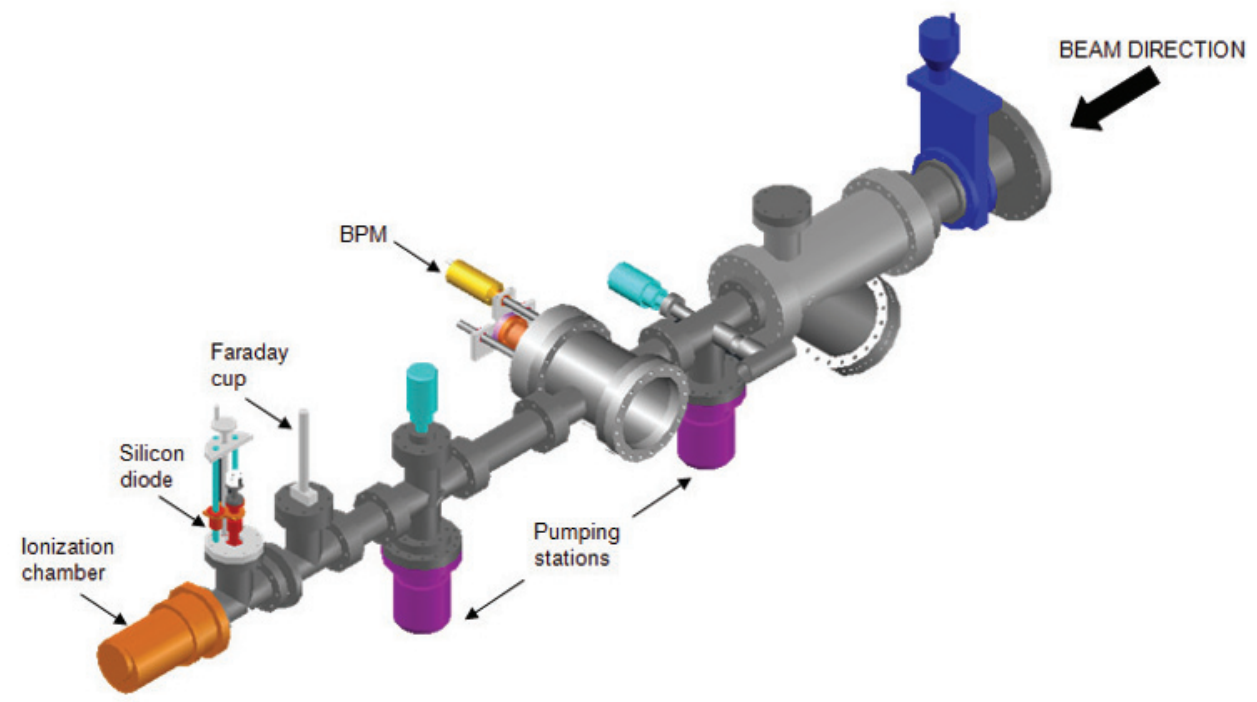

Figure 2: Mechanical drawing of the final part of the AMS beam line, downstream the high-energy side electrostatic analyser.

tional chamber used as a Beam Profile Monitor (BPM) for very low intensity ion beams [10] and a silicon diode used as the rare isotope detector. Actually, this silicon detector is mounted on a retractable arm, thus allowing us to remove it from the ions path and then use the pre-existing ionisation chamber to count the ${ }^{14} \mathrm{C}$ particles.

Typical achievable precision is better than 5\%o and 3\%o for measured ${ }^{14} \mathrm{C} /{ }^{12} \mathrm{C}$ and ${ }^{13} \mathrm{C} /{ }^{12} \mathrm{C}$ isotopic ratios, respectively. During each beam run, accuracy is routinely monitored by measuring samples prepared from standard reference materials, whose ${ }^{14} \mathrm{C}$ concentration is certified, and normalizing them to our primary standard, NIST HOxII. Figure 3 shows experimental data of the IAEA-C7 samples (IAEA, International Agency of Atomic Energy) measured at LABEC since mid 2004, when the accelerator began to be fully operative. In the graph, the measured ${ }^{14} \mathrm{C}$ concentration is reported as pMC (percent of Modern Carbon): AMS-measured ${ }^{14} \mathrm{C} /{ }^{12} \mathrm{C}$ isotopic ratios have been corrected for isotopic fractionation $\left({ }^{13} \mathrm{C} /{ }^{12} \mathrm{C}\right.$ ratios also measured in the same run in the accelerator) and for background, and normalized to the isotopic ratios measured for the NIST HOxII samples. Data are reported with 1- $\sigma$ error bars. The agreement with the certified value ( $49.53 \pm 0.12 \mathrm{pMC}$, see the dotted line in the graph) is satisfactory, showing the good performance of the LABEC ${ }^{14} \mathrm{C}$-AMS set-up. 


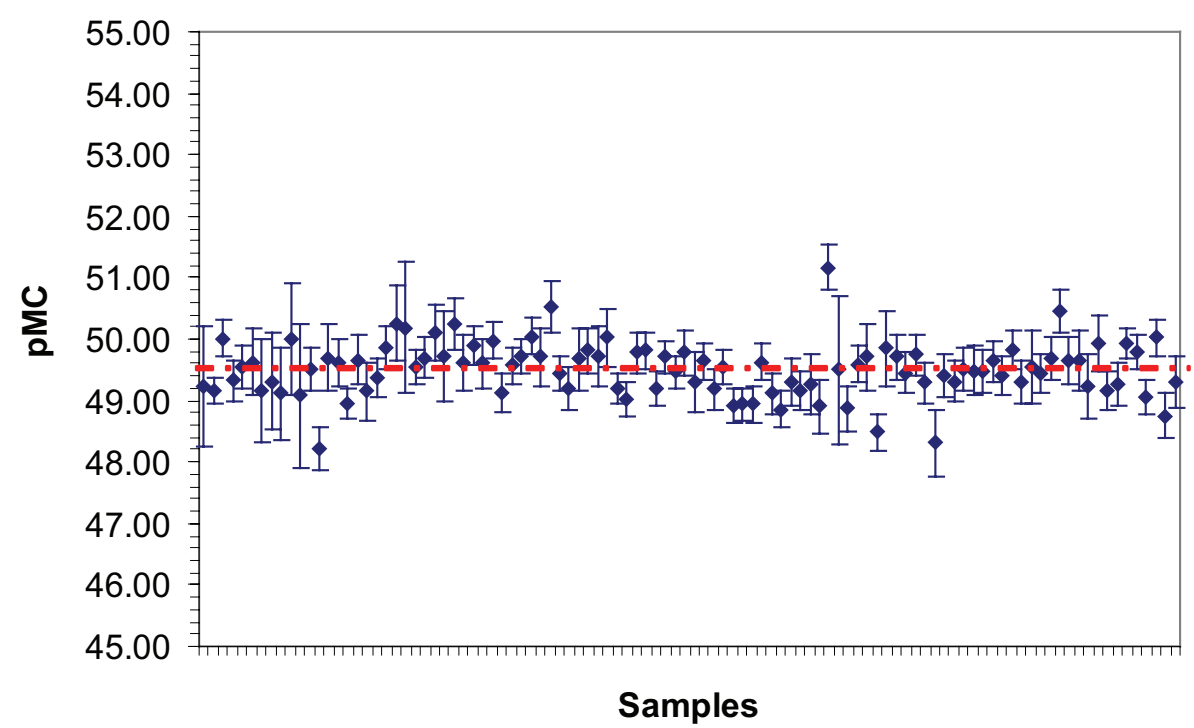

Figure 3: IAEA-C7 samples measured at LABEC since mid 2004, when the accelerator began to be fully operative.

\section{Dating foraminifera to estimate sedimentation rates and study marine archives}

The study of marine archives is usually performed by collecting sediment cores, few meters long, which are then divided in several slices. Each slice is characterised by physical and chemical properties that are specific of a particular timing period. Instead of ${ }^{14} \mathrm{C}$-dating bulk organic-rich sediments, i.e. just the bulk organic matter dispersed in the sediment matrix, which can be a mixture of different sources, dating biogenic carbonates is preferable. In the case of deep-sea sediments, foraminifera (forams) are usually the targets. They are typically handpicked from sediments and dated [11].

Foraminifera are unicellular organisms that live in the oceans, either in the water column (planktonic species) or in the seafloor (benthic species); during their life, many of them produce a calcium carbonate precipitate like a shell, called test, while others made their tests agglutinating sediment particles. They are usually less than $1 \mathrm{~mm}$, even down to a few tens of microns. For radiocarbon measurements, when possible, monospecific samples are collected and planktonic foraminifera are usually selected. Radiocarbon concentrations in planktonic foraminifera reflect the ${ }^{14} \mathrm{C}$ concentration in 
the water column at the depth where these organisms have lived; in fact, after their death, they fall onto the sea floor, during a time range from 3 days to 2 weeks, and then they are "stored" in the sedimentary record. Taking into account that the ${ }^{14} \mathrm{C}$ concentrations in the forams are affected by the reservoir effect, experimental ${ }^{14} \mathrm{C}$ data have to be corrected for reservoir age.

Considering that foraminifera are tiny and cannot be so abundant in sediments, it is evident that in each slice of sediment only few mg of samples can be collected at the maximum. Before dating, each sample has to be purified and cleaned in order to remove any possible source of external carbon that might contaminate the original radiocarbon concentration. Some of these undesirable components are clays, dust, secondary carbonates, organic matter and/or adsorbed atmospheric $\mathrm{CO}_{2}$. As far as carbonate samples are concerned, a typical procedure is based on $\mathrm{HCl}$ etching of samples to remove the most external layers, up to $30 \%$ by weight [12]. Being the foraminifera tests more delicate than shell "macrosamples", a more delicate approach is preferable [13].

At LABEC, forams are treated with hydrogen peroxide in a ultrasonic bath for about 10 minutes: $\mathrm{H}_{2} \mathrm{O}_{2}$ is slightly acidic, thus it produces a mild leach of the external surface of the samples, without causing too much a decrease in sample mass. After washing, samples are rinsed with deionised water for several times until $\mathrm{pH}$ neutrality is reached. Then, they are vacuum dried at $35^{\circ} \mathrm{C}$ overnight. A low temperature is chosen in order to minimize the re-adsorption of atmospheric $\mathrm{CO}_{2}$ onto the cleaned carbonate surfaces.

Instead of extracting $\mathrm{CO}_{2}$ from purified foraminifera by hydrolysis with phosphoric acid (as using the typical procedure found in the literature, see for example [14]), at LABEC, collection of $\mathrm{CO}_{2}$ is achieved by burning the samples in a CHN elemental analyser (ThermoFlash EA 1112), directly coupled to a graphitisation line where the carbon dioxide is converted into elemental carbon (graphite). Few $\mathrm{mg}$ of the cleaned and dried samples $(\sim 4.5 \mathrm{mg})$ are put in small tin capsules and inserted into the elemental analyser. Inside it, the different gases evolving from the combustion, i.e. $\mathrm{CO}_{2}$ and $\mathrm{H}_{2} \mathrm{O}$ in this case, are separated by passing through a gas-chromatographic column. The outlet of the elemental analyser is then connected to a three-way valve, which allows us to open the connection to the graphitisation line during the time interval $\mathrm{CO}_{2}$ is flowing out and to let the other gases exhausting into the atmosphere. Once the $\mathrm{CO}_{2}$ is collected in any of the four installed reaction chambers, conversion to graphite is achieved by the so-called Bosh reaction [15]:

$$
\mathrm{CO}_{2}+2 \mathrm{H}_{2} \stackrel{\mathrm{Fe}, 600^{\circ} \mathrm{C}}{\longrightarrow} \mathrm{C}+2 \mathrm{H}_{2} \mathrm{O},
$$



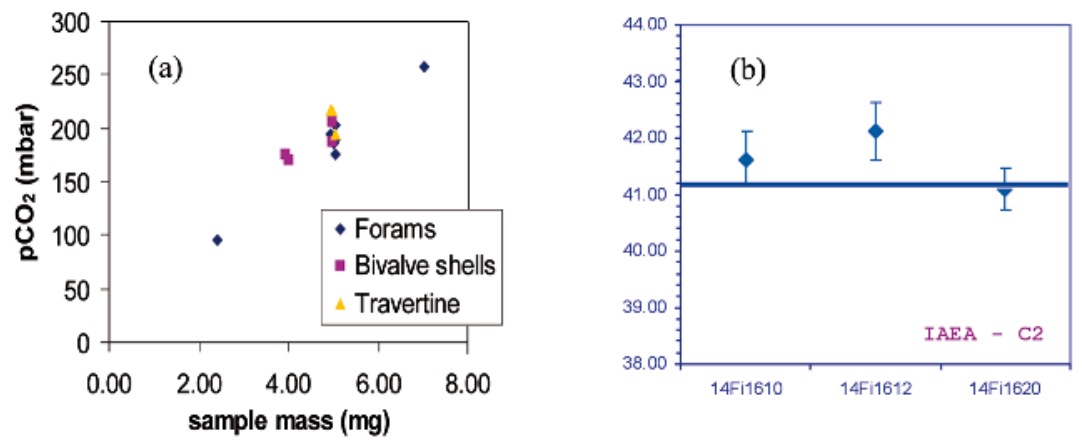

Figure 4: (a): $\mathrm{CO}_{2}$ collected (pressure measured in the graphitization reactor) as a function of the combusted sample mass; (b): radiocarbon concentration measured for three IAEA-C2 samples (standard certified value is indicated by the dotted line).

where iron powder (Alfa Aesar ${ }^{\circledR}, 99.9+\%,<10 \mu \mathrm{m}$ ) is used as the catalyst. Typical sample sizes are $\sim 400 \mu \mathrm{g}$ of carbon.

The overall process has been tested for carbonate samples, verifying both the reproducibility of the combustion in the elemental analyser and the accuracy of the ${ }^{14} \mathrm{C}-\mathrm{AMS}$ measurement. Figure 4 (a) shows the amount of $\mathrm{CO}_{2}$ collected as a function of the combusted sample mass: the linear behaviour suggests a good reproducibility of the combustion. Figure 4 (b) shows experimental data of three IAEA-C2 samples. IAEA-C2 is travertine, the reference material selected for carbonates. In the graph, data are reported with 1- $\sigma$ error bars. Although only three samples have been prepared and measured so far, the agreement with the certified value $(41.14 \pm 0.03 \mathrm{pMC})$ is quite satisfactory (see the dotted line in the graph).

\section{Measuring ${ }^{14} \mathrm{C}$ in particulate matter for source apportionment studies}

Carbon is a major constituent of particulate matter in the atmospheric aerosols [16]. It is present in the aerosol either with a graphitic microstructure or as organic compounds; therefore, the total carbon (TC) present in the aerosol can be divided into elemental carbon (EC) and organic carbon (OC). EC and OC have very different chemical and optical properties. Without going into the details, the EC is emitted in combustion processes and is light-absorbing. The OC may be emitted in combustion processes or by natural sources, but can also be formed in the atmosphere as secondary aerosol through gas-phase photochemical processes; it is mainly light-scattering. 
Actually, carbon may be present in atmosphere as carbonate too (from the resuspension of crustal material containing carbonate species); however, this contribution is mostly negligible [16].

Radiocarbon measurements have been demonstrated as a powerful tool to study the sources of the carbonaceous aerosol fraction [17]. Based on a simple two-sources model, it is possible to distinguish and quantify the contributions from the combustion of fossil fuels from the other contributions, i.e. from those due to biogenic sources and to biomass burning. As already anticipated in the introduction, fossil fuels are so old that can be considered radiocarbon-free, while the other two sources are modern and are characterized by almost the same radiocarbon concentration as the present atmosphere.

A further improvement in carbonaceous particles source apportionment is possible when radiocarbon measurements are performed on the sample carbonaceous sub-fractions EC and OC. In fact, the EC is emitted only in combustion processes, either of fossil fuels or biomasses. Therefore, the application of the simple two-sources model just on this EC fraction allows us to distinguish and quantify the contributions due to the fossil fuel combustion and to the biomass burning. As far as the OC is concerned, the contribution due to the biomass burning source may be derived by the contribution that this source gives to EC, when the OC/EC emission ratio is known; by this way, a complete source apportionment among the three OC sources (fossil fuel combustion, biomass burning and biogenic source) is possible.

To be analysed for TC/EC/OC and radiocarbon, the aerosol has to be collected on quartz fibre filters. These filters do not contain carbon and can sustain the high temperatures needed for these analyses. The collected mass depends on the kind of sampler used, on the collection time and on the atmospheric concentration. Typical concentrations on our filters are mainly in the range of $120-140 \mu \mathrm{g} / \mathrm{cm}^{2}$ (for PM10 samples) and carbon represents up to $50 \%$ of the total PM mass.

In order to perform the radiocarbon measurements on the separate carbonaceous sub-fractions, a suited sample preparation line was realized at LABEC. Such dedicated sample preparation line was needed in order to process the small quantities of carbon available in the particulate matter samples $(\ll 1 \mathrm{mg})$ and to fulfil all the requirements for the EC/OC separation. This line is essentially based on four basic parts: a first one for the cleaning of the carrier gases; a second one dedicated to the combustion of the samples; a third one devoted to the selection and trapping of the produced $\mathrm{CO}_{2}$; in the last part, the trapped $\mathrm{CO}_{2}$ is converted into graphite, in 


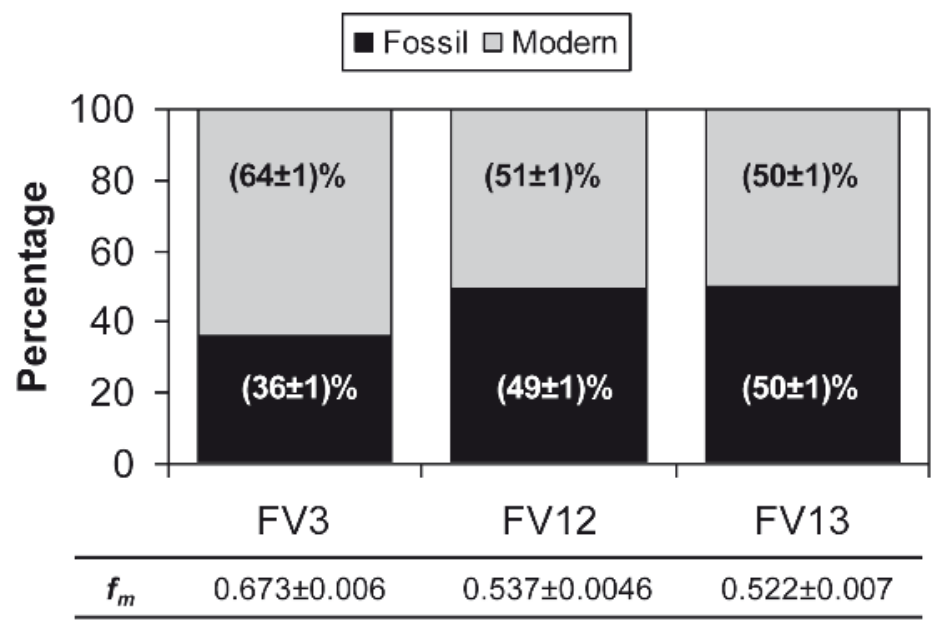

Figure 5: Aerosol samples collected in Milan during the summer period: measured radiocarbon concentration, as $f_{m}$, and corresponding source apportionment based on the two-sources model are reported.

order to be pressed into the sample-holder capsules. The line is described in detail in [17]. So far, graphite samples of $110-450 \mu \mathrm{g}$ of carbon have been prepared.

The TC measurement protocol was fully tested by measuring standards and blanks (i.e. samples with nominally no ${ }^{14} \mathrm{C}$ ); this protocol is based on a 20 minutes-long combustion at $800^{\circ} \mathrm{C}$ with a $100 \mathrm{~cm}^{3} \mathrm{~min}^{-1} \mathrm{O}_{2}$ flow. The efficiency of the new combustion-graphitization line was measured to be very high $(\sim 100 \%)$.

Some measurements on real samples have been already performed on aerosol samples collected in Milan during the summer period. In Figure 5 , the measured radiocarbon concentrations are reported as $f_{m}$ (fraction of modern, the unit typically used in this kind of applications, basically $0.01 \mathrm{pMC}$ ); a source apportionment according to the two-source model is also shown (see the discussion at the beginning of this paragraph). For this apportionment, we assumed $f_{m, \bmod }=1.055 \pm 0.015$ [18].

\section{Conclusion and outlook}

The discussion above has shown how important radiocarbon can be in solving environmental issues both in the past and in the present. In particular, the attention has been focused on the hardware and on the procedures 
developed at INFN-LABEC to study marine environments, by radiocarbon dating of foraminifera tests, and atmospheric aerosols, by ${ }^{14} \mathrm{C}$ measurements of carbonaceous fractions in particulate matter samples.

Preliminary results obtained by dating foraminifera from coastal marine sediments of the NW Spanish coast have proven to be reliable data that will be integrated with sediment proxies to discuss environmental changes in this area for the last millennia.

As far as aerosol measurements are concerned, the TC protocol will be soon applied in specific sampling campaigns focused on the carbonaceous aerosol source apportionment; in addition, protocols that are suited for EC/OC separations are currently under investigation.

\section{Acknowledgments}

We would like to thank Prof. P.A. Mandò for the fruitful discussion. The activity described here has been developed thanks to the support of INFN, through the experiments RIDAGMA and NUMEN, funded by the V National committee. Work on particulate matter radiocarbon measurements has been also funded by the Ministry of Education, University, and Research under the PRIN2007 grant. The financial support of Regione Toscana (in the framework of the TEMART project - POR CReO/FESR 2007-2013) is gratefully acknowledged for the position of G. Calzolai within the INFN.

\section{References}

[1] Godwin H. Nature 195 (1962) 894.

[2] Rasmussen T. L., Thomsen E., Troelstra S. R., Kuijpers A., Prins A. Mar. Micropaleontol. 47 (2002) 143.

[3] Peterson L. C., Haug G. H., Hughen K. A., Röhl U. Science 290 (2000) 1947.

[4] Forster P., Ramaswamy V., Artaxo P., Berntsen T., Betts R., Fahey D. W., Haywood J., Lean J., Lowe D. C., Myhre G., Nganga J., Prinn R., Raga G., Schulz M., Van Dorland R., Changes in Atmospheric Constituents and in Radiative Forcing. In: Climate Change 2007: The Physical Science Basis. Contribution of Working Group I to the Fourth Assessment Report of the Intergovernmental Panel on Climate Change, edited by Solomon, S., D. Qin, M. Manning, Z. Chen, M. 
Marquis, K. B. Averyt, M. Tignor and H. L. Miller (Cambridge University Press, Cambridge, United Kingdom and New York, NY, USA) 2007.

[5] Smith A. M., Hua Q., Williams A., Levchenko V., Yang B., Nucl. Instrum. Methods B 268 (2010) 919.

[6] Fedi M. E., Cartocci A., Manetti M., Taccetti F. and Mandò P. A., Nucl. Instrum. Methods B 259 (2007) 18.

[7] Mandò P. A., Nuclear Physics News 19 (2009) 5.

[8] Calzolai G., Chiari M., García Orellana I., Lucarelli F., Migliori A., Nava S., Taccetti F., Nucl. Instrum. Methods B 249 (2006) 928.

[9] Mirto F. A., Carraresi L. Nucl. Instrum. Methods B 266 (2008) 2113.

[10] Taccetti F., Carraresi L., Fedi M. E., Manetti M., Mariani P., Tobia G., Mandò P. A., Radiocarbon 52 (2010) 272.

[11] Bond G., Showers W., Cheseby M., Lotti R., Almasi P., de Menocal P., Priore P., Cullen H., Hajdas I., Bonani G., Science 278 (1997) 1257.

[12] Soares A. M. M., Dias J. M. A., Radiocarbon 48 (2006) 45.

[13] Schleicher M., Grootes P., Nadeau M. J., Schoon A., Radiocarbon 40 (1998) 85.

[14] McNichol A. P., Osborne E. A., Gagnon A. R., Fry B., Jones G. A., Nucl. Instrum. Methods B 92 (1994) 162.

[15] Vogel J. S., Southon J. R., Nelson D. E., Brown T. A., Nucl. Instrum. Methods B 5 (1984) 289.

[16] ten Brink H., Maenhaut W., Hitzenberger R., Ganuk T., Splinder G., Even A., Chi X., Bauer H., Puxbaum H., Putaud J.-P., Tursic J., Berner A., Atm. Env. 38 (2004) 6507.

[17] Currie L. A., Radiocarbon 42 (2000) 115.

[18] Calzolai G., Bernardoni V., Chiari M., Fedi M. E., Lucarelli F., Nava S., Riccobono F., Taccetti F., Valli G., Vecchi R., Nucl. Instrum. Methods B 269 (2011) 203. 
[19] Szidat S., Ruff M., Perron N., Wacker L., Synal H.-A., Hallquist M., Shanningrahi A. S., Yttri K. E., Dye C., Simpson D., Atmos. Chem. Phys. 9 (2009) 1521. 